\title{
PERSISTENCE AND EQUILIBRIA OF BRANCHING POPULATIONS WITH EXPONENTIAL INTENSITY
}

\author{
ZAKHAR KABLUCHKO, ${ }^{*}$ Ulm University
}

\begin{abstract}
We consider a system of independent branching random walks on $\mathbb{R}$ which start from a Poisson point process with intensity of the form $e_{\lambda}(\mathrm{d} u)=\mathrm{e}^{-\lambda u} \mathrm{~d} u$, where $\lambda \in \mathbb{R}$ is chosen in such a way that the overall intensity of particles is preserved. Denote by $\chi$ the cluster distribution, and let $\varphi$ be the log-Laplace transform of the intensity of $\chi$. If $\lambda \varphi^{\prime}(\lambda)>0$, we show that the system is persistent, meaning that the point process formed by the particles in the $n$th generation converges as $n \rightarrow \infty$ to a nontrivial point process $\Pi_{e_{\lambda}}^{\chi}$ with intensity $e_{\lambda}$. If $\lambda \varphi^{\prime}(\lambda)<0$ then the branching population suffers local extinction, meaning that the limiting point process is empty. We characterize point processes on $\mathbb{R}$ which are cluster invariant with respect to the cluster distribution $\chi$ as mixtures of the point processes $\Pi_{c e_{\lambda}}^{\chi}$ over $c>0$ and $\lambda \in K_{\text {st }}$, where $K_{\text {st }}=\{\lambda \in \mathbb{R}: \varphi(\lambda)=0$, $\left.\lambda \varphi^{\prime}(\lambda)>0\right\}$.
\end{abstract}

Keywords: Branching random walk; Poisson point process; exponential intensity; persistence; local extinction; equilibrium state; cluster invariant point process

2010 Mathematics Subject Classification: Primary 60J80

Secondary 60G55

\section{Introduction}

\subsection{Persistence criterion}

We consider a population of particles on $\mathbb{R}^{d}$ whose stochastic evolution is governed by the following rules. The initial positions of the particles form a Poisson point process on $\mathbb{R}^{d}$ denoted by $\pi_{0}$. The intensity measure of $\pi_{0}$, denoted by $v$, is assumed to be finite on bounded sets. After one unit of time each particle is replaced, independently of all other particles, by a random cluster of offspring whose displacements with respect to the parent particle are governed by a point process $\chi$ (which is allowed to be empty with positive probability). Note that the distribution of $\chi$ does not depend on the position of the parent particle. The daughter particles form a point process denoted by $\pi_{1}$. Then, any of the daughter particles is independently replaced by a random cluster of granddaughter particles, forming a point process $\pi_{2}$, and so on. We denote the point process formed by the particles of the $n$th generation by $\pi_{n}$. We will be interested in the behavior of $\pi_{n}$ as $n \rightarrow \infty$.

This problem has been much studied, especially in the case when the cluster distribution $\chi$ is critical (i.e. the mean number of particles in the cluster $\chi$ is 1) and $v$ is the Lebesgue measure; see [11], [12], [15], [21, Chapters 11, 12], and [24]. In this particular case it has been shown, under a second moment condition on the intensity of the clusters, that in dimensions $d=1$ and $d=2$ the branching population suffers local extinction. This means that the point process $\pi_{n}$

Received 1 April 2011; revision received 18 October 2011.

* Postal address: Institute of Stochastics, Ulm University, Helmholtzstrasse 18, 89069 Ulm, Germany.

Email address: zakhar.kabluchko@uni-ulm.de 
converges as $n \rightarrow \infty$ to the empty point process. In dimension $d \geq 3$, the branching population is persistent, meaning that $\pi_{n}$ converges to some nontrivial point process whose intensity is equal to the Lebesgue measure. A general theory of convergence to equilibrium of branching populations on an arbitrary Polish space was developed in [17]; see also [23].

We will be interested what happens if the Poisson point process $\pi_{0}$ formed by the initial positions of the particles is not homogeneous, i.e. the measure $v$ is not the Lebesgue measure. Let $J$ be the intensity of the point process $\chi$, that is, $J$ is a measure on $\mathbb{R}^{d}$ given by $J(B)=\mathrm{E}[\chi(B)]$ for every Borel set $B \subset \mathbb{R}^{d}$. We make the following moment assumption:

$$
\mathrm{E}\left[\chi\left(\mathbb{R}^{d}\right) \int_{\mathbb{R}^{d}} \mathrm{e}^{\langle t, u\rangle} \chi(\mathrm{d} u)\right]<\infty, \quad t \in \mathbb{R}^{d} .
$$

Here $\langle\cdot, \cdot\rangle$ denotes the scalar product on $\mathbb{R}^{d}$. In particular, the log-Laplace transform of $J$ is finite:

$$
\varphi(t):=\log \int_{\mathbb{R}^{d}} \mathrm{e}^{\langle t, u\rangle} J(\mathrm{~d} u)<\infty, \quad t \in \mathbb{R}^{d} .
$$

When studying persistence of a branching population, it is natural to require that the intensity of the point process $\pi_{n}$ should remain constant in time. This leads to a convolution equation

$$
J * v=v .
$$

The solutions of this equation have been described by Deny [10]. For $\lambda \in \mathbb{R}^{d}$, let $e_{\lambda}$ be a measure on $\mathbb{R}^{d}$ whose density with respect to the Lebesgue measure is given by

$$
e_{\lambda}(\mathrm{d} u)=\mathrm{e}^{-\langle\lambda, u\rangle} \mathrm{d} u .
$$

An easy computation shows that any measure of the form $e_{\lambda}$, where $\lambda$ is such that $\varphi(\lambda)=0$, is a solution to (1.3). Conversely, under a nonlattice assumption on $J$, Deny [10] showed that any measure $v$ which solves (1.3) and is finite on bounded sets can be represented as a mixture of the form

$$
v(\cdot)=\int_{K} e_{\lambda}(\cdot) \mu(\mathrm{d} \lambda)
$$

where $\mu$ is a finite measure on the set $K:=\left\{\lambda \in \mathbb{R}^{d}: \varphi(\lambda)=0\right\}$.

In the next theorem we give a criterion for persistency/local extinction of the branching population starting from a Poisson process with intensity measure $e_{\lambda}, \lambda \in K$. Denote by $\nabla$ the gradient operator.

Theorem 1.1. Consider a branching population satisfying (1.1) which starts as a Poisson point process with intensity $e_{\lambda}$, where $\lambda \in \mathbb{R}^{d}$ is such that $\varphi(\lambda)=0$.

1. If $\langle\lambda, \nabla \varphi(\lambda)\rangle>0$ then the point process $\pi_{n}$ converges weakly to some point process $\Pi_{e_{\lambda}}^{\chi}$ having intensity $e_{\lambda}$ (the branching population is persistent).

2. If $\langle\lambda, \nabla \varphi(\lambda)\rangle<0$ then the point process $\pi_{n}$ converges weakly to the empty point process (the branching population suffers local extinction).

\subsection{The one-dimensional case}

It is instructive to consider the special case $d=1$; see Figure 1 . To exclude trivialities, we assume that $J$ is not concentrated on $[0,+\infty)$ or $(-\infty, 0]$. This implies that $\lim _{t \rightarrow \pm \infty} \varphi(t)=$ $+\infty$. The function $\varphi$ is strictly convex. 


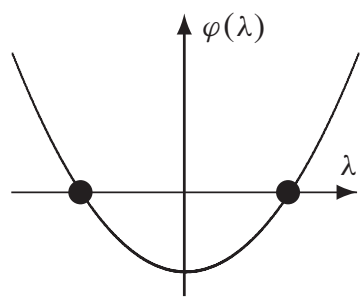

(a)

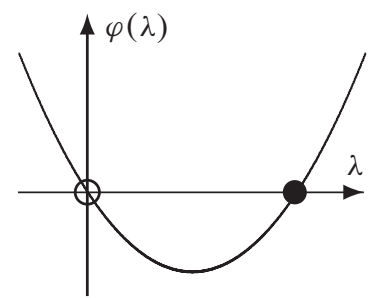

(b)

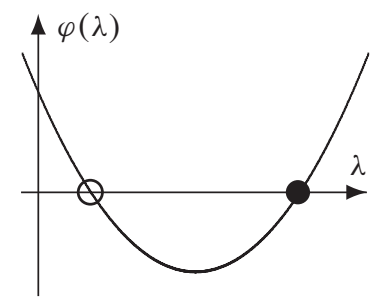

(c)

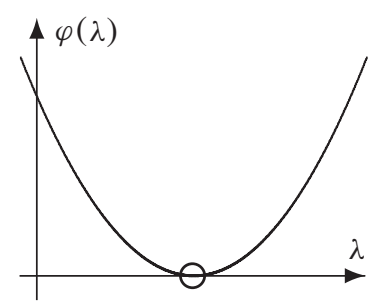

(d)

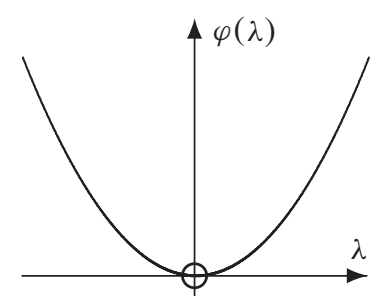

(e)

Persistence Local extinction

FIGURE 1: Persistence versus local extinction of one-dimensional branching populations: (a) subcritical case; (b) critical case; (c) supercritical case; (d) supercritical, boundary case; (e) critical, boundary case.

Assume first that the cluster distribution $\chi$ is subcritical in the sense that the mean number of offspring of any particle is less than 1 . Note that this implies that the progeny of any individual particle dies out with probability 1 ; see [3, p. 7]. Let us see what happens to the population as a whole. Since the subcriticality means that $\varphi(0)<0$, the equation $\varphi(\lambda)=0$ has two solutions, $\lambda_{1}<0$ and $\lambda_{2}>0$, which satisfy $\varphi^{\prime}\left(\lambda_{1}\right)<0$ and $\varphi^{\prime}\left(\lambda_{2}\right)>0$. By Theorem 1.1 , both measures $e_{\lambda_{1}}$ and $e_{\lambda_{2}}$ give rise to a persistent branching population. In both cases we have a system of branching random walks which become extinct individually but survive as a collective.

Assume now that the cluster distribution $\chi$ is critical, that is, the mean number of offspring of any particle is 1 . Equivalently, $\varphi(0)=0$. Again, the progeny of any individual particle dies out with probability 1 ; see [3, p. 7]. If $\varphi^{\prime}(0)=\int_{\mathbb{R}} u J(\mathrm{~d} u)=0$ then the equation $\varphi(\lambda)=0$ has only one solution, $\lambda=0$. It is known, see [15], that the corresponding branching population, starting with the Lebesgue measure, suffers local extinction. We now assume that $\int_{\mathbb{R}} u J(\mathrm{~d} u)<0$, which means that the particles have a drift to the left. (The case $\int_{\mathbb{R}} u J(\mathrm{~d} u)>0$ is analogous.) Then, $\varphi(0)=0$ and $\varphi^{\prime}(0)<0$. Hence, the equation $\varphi(\lambda)=0$ has two solutions, $\lambda_{1}=0$ and $\lambda_{2}>0$. By [15], the first solution $\lambda_{1}=0$ gives rise to a branching population which suffers local extinction. However, by Theorem 1.1, the second solution $\lambda_{2}$ corresponds to a persistent branching population. Thus, a critical branching population can survive provided that the particles have a nonzero drift and they have appropriately chosen their starting positions.

Assume finally that the cluster distribution $\chi$ is supercritical, i.e. the mean number of offspring of any particle is greater than 1 . Equivalently, $\varphi(0)>0$. There are three possibilities. First, it may happen that the equation $\varphi(\lambda)=0$ has no solutions, which is not interesting to us. Second, it may happen that the equation $\varphi(\lambda)=0$ has two different solutions of the same sign. By Theorem 1.1, the solution which is at a greater distance from 0 gives rise to a persistent branching population, whereas the branching population corresponding to the remaining solution becomes locally extinct. Thus, a supercritical branching population may suffer local extinction, even though the progeny of any particle survives with positive 
probability and grows exponentially on survival. Finally, it may happen that the equation $\varphi(\lambda)=0$ has only one solution $\lambda_{1}$ such that $\varphi^{\prime}\left(\lambda_{1}\right)=0$. This supercritical boundary case is not covered by Theorem 1.1. In Section 2.4 we will state that the branching population dies out in the supercritical boundary case if the underlying branching process is the binary branching Brownian motion, and conjecture that this holds for general branching random walks. The supercritical boundary case has been an object of recent interest due to its connection to the extremal process of the branching Brownian motion; see [1], [2], [8], [9], and [16].

A very simple special case arises if the cluster $\chi$ consists almost surely (a.s.) of exactly one particle (no branching). The persistence of the system is evident, since $\pi_{n}$ is a Poisson process with the same intensity $e_{\lambda}$ for every $n$. See [7], [13], [14], and [22] for various types of exponential intensity particle systems without branching.

\section{Properties of the branching population}

In this section we study the properties of the branching population in the one-dimensional case $d=1$. As it turns out, the picture of a persistent branching population is that of a system of individual progenies that (due to an entropic effect) 'invade' for a while in a direction which is opposite to the direction of the drift, eventually 'turn round', and finally 'wander off' in the direction of the drift. This type of behavior is reminiscent of the behavior of the extremes of a family of independent stochastic processes; see [7], [13], [14], and [22]. In a population suffering local extinction the entropic effect is too weak to enable invasion and the whole population wanders off in a uniform way at an asymptotically constant speed.

\subsection{Notation}

Let $\mathbb{M}$ be the space of locally finite counting measures (equivalently, discrete point configurations) on $\mathbb{R}$. A point process is a random element of $\mathbb{M}$. Given a point configuration $\rho=$ $\sum_{i \in \mathcal{I}} \delta_{V_{i}} \in \mathbb{M}$, where the index set $\mathcal{I}$ is at most countable, we denote by $\max \rho:=\max _{i \in \mathcal{I}} V_{i}$ the position of the rightmost atom (the maximal particle) of $\rho$ (provided that it exists). Also, for $u \in \mathbb{R}$, we denote by $T_{u} \rho=\sum_{i \in \ell} \delta_{u+V_{i}}$ the point configuration obtained by shifting every atom $V_{i}$ of $\rho$ by $u$. Let $\mathbb{N}_{0}=\{0,1, \ldots\}$ be the set of nonnegative integers.

We fix some point process $\chi$ (the distribution of clusters) with intensity $J$ satisfying (1.1). Note that the number of particles in $\chi$ is a.s. finite. A branching random walk with cluster distribution $\chi$ is a process which starts at time 0 with a single particle located at the origin and in which every unit of time every particle is independently replaced by a cluster of daughter particles whose displacements with respect to the parent particle are governed by the point process $\chi$. We denote by $\chi_{n}$ the point process formed by the particles in the $n$th generation of the branching random walk. By the Hammersley-Kingman-Biggins theorem [4], [5], the maximum $\max \chi_{n}$ of a supercritical branching random walk $\chi_{n}$ satisfies

$$
\lim _{n \rightarrow \infty} \frac{1}{n} \max \chi_{n}=\beta_{0} \quad \text { a.s. on survival, }
$$

where $\beta_{0}$ is the largest zero of $I(z)=\sup _{t \in \mathbb{R}}(z t-\varphi(t))$, the Legendre-Fenchel transform of $\varphi$. Note that $I$ is allowed to take the value $+\infty$.

In the sequel, we denote by $\pi_{0}=\sum_{i=1}^{\infty} \delta_{U_{i}}$ a Poisson point process on $\mathbb{R}$ with intensity $e_{\lambda}(\mathrm{d} u)=\mathrm{e}^{-\lambda u} \mathrm{~d} u$. Using this notation, the positions of the particles in the $n$th generation of a branching population starting at $\pi_{0}$ form a point process

$$
\pi_{n}=\sum_{i=1}^{\infty} T_{U_{i}}\left(\chi_{n}^{(i)}\right),
$$


where $\left\{\chi_{n}^{(i)}: n \in \mathbb{N}_{0}\right\}, i \in \mathbb{N}$, are copies of the branching random walk $\left\{\chi_{n}: n \in \mathbb{N}_{0}\right\}$, with all objects being independent.

\subsection{The maximal particle}

Let us look at the progeny of an individual particle in the branching population $\left\{\pi_{n}: n \in \mathbb{N}_{0}\right\}$. We claim that even in the persistent case, the progeny of any particle with probability 1 either dies out or drifts to $+\infty$ or $-\infty$. Indeed, if the cluster distribution $\chi$ is subcritical or critical, then the progeny of any particle dies out a.s.; see [3, p. 7]. If the cluster distribution is supercritical then the progeny survives with positive probability. However, assuming for concreteness that $\lambda>0$ and recalling that $\varphi(\lambda)=0$, it is easy to see that the limit $\beta_{0}$ in (2.1) is negative. Hence, the progeny of any individual particle either dies out or drifts to $-\infty$ in a uniform way.

These considerations suggest that the maximal particle of a persistent branching population at time $n$, where $n$ is large, is most likely to be an offspring of some particle which was located close to $-\infty$ at time 0 (we assume that $\lambda>0$ here). The next proposition makes this precise. Let $i_{n}$ denote the (random) number of the particle which is the ancestor at time 0 of the maximal particle of the population at time $n$. That is, $i_{n}$ is such that $\max \pi_{n}=U_{i_{n}}+\max \chi_{n}^{\left(i_{n}\right)}$.

Proposition 2.1. Assume that $\varphi(\lambda)=0, \lambda>0$, and $\varphi^{\prime}(\lambda)>0$. Then,

$$
\lim _{n \rightarrow \infty} \frac{1}{n} U_{i_{n}}=-\varphi^{\prime}(\lambda)<0 \quad \text { a.s. }
$$

In the supercritical or critical case, let $\beta_{0}$ be the largest 0 of the Legendre-Fenchel transform $I$. In the subcritical case, we set $\beta_{0}=-\infty$. In the next proposition we show that the maximum of a supercritical branching population $\pi_{n}$ suffering local extinction converges to $-\infty$ with the same asymptotic linear speed $\beta_{0}$ as the most successful offspring of a single particle.

Proposition 2.2. Assume that $\varphi(\lambda)=0, \lambda>0$, and $\varphi^{\prime}(\lambda)<0$. Then,

$$
\lim _{n \rightarrow \infty} \frac{1}{n} \max \pi_{n}=\beta_{0}<0 \quad \text { a.s. }
$$

Let us also mention the following proposition which will be obtained as a simple corollary of Theorem 1.1.

Proposition 2.3. Let $\left\{\chi_{n}: n \in \mathbb{N}_{0}\right\}$ be a branching random walk with cluster distribution $\chi$ which satisfies (1.1). Let $t \geq 0$.

1. If $\varphi^{\prime}(t)>\beta_{0}$ then $c(t):=\lim _{n \rightarrow \infty} \mathrm{e}^{-\varphi(t) n} \mathrm{E}\left[\mathrm{e}^{t \max \chi_{n}}\right]$ exists in $(0, \infty)$.

2. If $\varphi^{\prime}(t)<\beta_{0}$ then $c(t):=\lim _{n \rightarrow \infty} \mathrm{e}^{-\varphi(t) n} \mathrm{E}\left[\mathrm{e}^{t \max \chi_{n}}\right]=0$.

Remark 2.1. We conjecture that, for $\varphi^{\prime}(t)=\beta_{0}$, the limit is 0 . The proof in the case of the binary branching Brownian motion will be given below; see (3.7).

The next proposition states that the maximal particle of the point process $\Pi_{e_{\lambda}}^{\chi}$ which appeared in Theorem 1.1 has a shifted Gumbel distribution.

Proposition 2.4. Assume that $\varphi(\lambda)=0, \lambda>0$, and $\varphi^{\prime}(\lambda)>0$. Let $c(\lambda)$ be as in Proposition 2.3. Then,

$$
\mathrm{P}\left[\max \Pi_{e_{\lambda}}^{\chi} \leq z\right]=\exp \left\{-\frac{c(\lambda)}{\lambda} \mathrm{e}^{-\lambda z}\right\}, \quad z \in \mathbb{R} .
$$




\subsection{Equilibrium states and their family structure}

A point process $\Xi$ on $\mathbb{R}$ is called an equilibrium state for the cluster distribution $\chi$ if the branching population starting at $\Xi$ is stationary in time. In other words, if $\sum_{i=1}^{\infty} \delta_{V_{i}}$ is a realization of $\Xi$ and the $\chi^{(i)}, i \in \mathbb{N}$, are copies of the point process $\chi$ (all objects being independent), then the point process $\sum_{i=1}^{\infty} T_{V_{i}} \chi^{(i)}$ has the same law as $\Xi$. Clearly, the point process $\Pi_{e_{\lambda}}^{\chi}$ is an equilibrium state for every $\lambda \in K_{\text {st }}$, where $K_{\text {st }}=\{\lambda \in \mathbb{R}: \varphi(\lambda)=0$, $\left.\lambda \varphi^{\prime}(\lambda)>0\right\}$. Moreover, let $\Pi_{c e_{\lambda}}^{\chi}$ be the limiting point process for the branching population started from the Poisson point process with intensity $c e_{\lambda}$, where $c>0$ and $\lambda \in K_{\text {st. Then, any }}$ mixture of the form

$$
\Xi(\cdot)=\int_{(0, \infty) \times K_{\mathrm{st}}} \Pi_{c e_{\lambda}}^{\chi}(\cdot) \mu(\mathrm{d} c, \mathrm{~d} \lambda),
$$

where $\mu$ is a probability measure on the space $(0, \infty) \times K_{\text {st }}$, is an equilibrium state. Note at this point that $K_{\text {st }}$ consists of at most two elements. The next theorem describes the set of equilibrium states in the nonboundary case. A related result for systems without branching can be found in [22]. Recall that a measure $J$ on $\mathbb{R}$ is called nonlattice if it is not concentrated on an arithmetic progression of the form $a \mathbb{Z}+b$, where $a, b \in \mathbb{R}$.

Theorem 2.1. Let $\chi$ be a cluster distribution satisfying (1.1) and having a nonlattice intensity measure J. Assume that the equation $\varphi(\lambda)=0$ has two different solutions. Then, any equilibrium state $\Xi$ whose intensity measure is finite on bounded sets can be represented as a mixture of the form (2.3).

Remark 2.2. In the case $d>1$ any equilibrium state $\Xi$ can be represented as a mixture of the form (2.3), where $K_{\mathrm{st}}$ should be defined as the set of those $\lambda \in K$ such that the branching population starting from a Poisson point process with intensity $e_{\lambda}$ is persistent. Recall that $K=\left\{\lambda \in \mathbb{R}^{d}: \varphi(\lambda)=0\right\}$. The question is how to describe the set $K_{\text {st }}$. If the cluster distribution $\chi$ is subcritical then $K$ is a convex surface enclosing the origin. It is easy to see that any $\lambda \in K$ satisfies $\langle\lambda, \nabla \varphi(\lambda)\rangle>0$ and, hence, by Theorem 1.1, $K_{\text {st }}=K$. Similarly, in the critical case, any $\lambda \in K \backslash\{0\}$ satisfies $\langle\lambda, \nabla \varphi(\lambda)\rangle>0$ and, hence, belongs to $K_{\text {st }}$. As for $\lambda=0$, it is known that it belongs to $K_{\text {st }}$ if and only if $d>2$; see [15]. In the supercritical case, any $\lambda \in K$ such that $\langle\lambda, \nabla \varphi(\lambda)\rangle>0$ belongs to $K_{\text {st }}$, any $\lambda \in K$ such that $\langle\lambda, \nabla \varphi(\lambda)\rangle<0$ does not belong to $K_{\text {st }}$. However, for $d>1$, we can always find $\lambda \in K$ with $\langle\lambda, \nabla \varphi(\lambda)\rangle=0$. Geometrically, such $\lambda$ s are the points where the tangent lines passing through the origin meet $K$. We do not know whether such $\lambda$ s belong to $K_{\text {st }}$ or not.

Consider a branching population $\left\{\tilde{\pi}_{n}: n \in \mathbb{N}_{0}\right\}$ starting with a point process $\Pi_{e_{\lambda}}^{\chi}$. That is, $\tilde{\pi}_{n}=\sum_{i=1}^{\infty} T_{V_{i}}\left(\chi_{n}^{(i)}\right)$, where $\sum_{i=1}^{\infty} \delta_{V_{i}}$ is a realization of $\Pi_{e_{\lambda}}^{\chi}$ and $\left\{\chi_{n}^{(i)}: n \in \mathbb{N}_{0}\right\}, i \in \mathbb{N}$, are copies of the branching random walk $\left\{\chi_{n}: n \in \mathbb{N}_{0}\right\}$ (all objects being independent). Being stationary, the $\mathbb{M}$-valued process $\left\{\tilde{\pi}_{n}: n \in \mathbb{N}_{0}\right\}$ admits a two-sided stationary extension denoted by $\left\{\tilde{\pi}_{n}: n \in \mathbb{Z}\right\}$. Let $\mathcal{F}_{\leq n}$ be the $\sigma$-algebra generated by the random variables $\tilde{\pi}_{k}(B)$, where $B \subset \mathbb{R}$ is a bounded interval and $k=n, n-1, \ldots$ The $\sigma$-algebra of the infinite past $\mathcal{F}_{-\infty}$ is defined by $\mathcal{F}_{-\infty}=\bigcap_{n \in \mathbb{Z}} \mathcal{F}_{\leq n}$.

Proposition 2.5. Assume that $\varphi(\lambda)=0$ and $\lambda \varphi^{\prime}(\lambda)>0$. Then, the $\sigma$-algebra $\mathcal{F}_{-\infty}$ is trivial, that is, every event in $\mathcal{F}_{-\infty}$ has probability 0 or 1 . As a consequence, the process $\left\{\tilde{\pi}_{n}: n \in \mathbb{Z}\right\}$ is ergodic and mixing.

Clearly, the union of two independent point processes distributed as $\Pi_{c_{1} e_{\lambda}}^{\chi}$ and $\Pi_{c_{2} e_{\lambda}}^{\chi}$ has the same law as $\Pi_{\left(c_{1}+c_{2}\right) e_{\lambda}}^{\chi}, c_{1}, c_{2}>0$. It follows that the point process $\Pi_{e_{\lambda}}^{\chi}$ is infinitely divisible; 
see [21, Section 1.6]. Moreover, the point process $\Pi_{e_{\lambda}}^{\chi}$ is superposable in the following sense; see [20]. Recall that $T_{u}$ denotes a translation by $u \in \mathbb{R}$ acting on the space $\mathbb{M}$ of locally finite counting measures on $\mathbb{R}$. Let $\Pi^{\prime}$ and $\Pi^{\prime \prime}$ denote two independent copies of $\Pi_{e_{\lambda}}^{\chi}$. Then, for every $u_{1}, u_{2}, u \in \mathbb{R}$ such that $\mathrm{e}^{\lambda u_{1}}+\mathrm{e}^{\lambda u_{2}}=\mathrm{e}^{\lambda u}$, we have the following equality in distribution:

$$
T_{u_{1}} \Pi^{\prime}+T_{u_{2}} \Pi^{\prime \prime} \stackrel{\mathrm{D}}{=} T_{u} \Pi_{e_{\lambda}}^{\chi} .
$$

To see that (2.4) holds, note that the Poisson point process with intensity $e_{\lambda}$ satisfies (2.4), and that superposability is preserved under clustering and taking weak limits. Superposable point processes have been characterized in [20]. As a consequence of Corollary 5.2 of [20], we obtain the following result.

Proposition 2.6. Assume that $\varphi(\lambda)=0, \lambda>0$, and $\varphi^{\prime}(\lambda)>0$. There is a unique in law point process $\Gamma$ (depending on $\lambda$ ) satisfying $\max \Gamma=0$ a.s. and a constant $c>0$ such that if the $\Gamma_{i}$, $i \in \mathbb{N}$, are independent copies of $\Gamma$ and, independently, $\sum_{i=1}^{\infty} \delta_{W_{i}}$ is a Poisson point process on $\mathbb{R}$ with intensity $c e_{\lambda}$, then

$$
\Pi_{e_{\lambda}}^{\chi} \stackrel{\mathrm{D}}{=} \sum_{i=1}^{\infty} T_{W_{i}} \Gamma_{i} .
$$

It is convenient to think of (2.5) as the decomposition of the point process $\Pi_{e_{\lambda}}^{\chi}$ into the families $T_{W_{i}} \Gamma_{i}, i \in \mathbb{N}$; see [17, Section 1.6]. If we continue the branching population starting with $\Pi_{e_{\lambda}}^{\chi}$ back into the past then any two particles alive at time 0 belong to the same family $T_{W_{i}} \Gamma_{i}$ if and only if they have a common ancestor somewhere in the past.

Proposition 2.7. Assume that $\varphi(\lambda)=0, \lambda>0$, and $\varphi^{\prime}(\lambda)>0$. Then, the point process $\Gamma$ consists of finitely many particles a.s. if and only if the cluster distribution $\chi$ is subcritical or $\mathrm{P}[\chi(\mathbb{R})=1]=1$.

We have already noted that even in the persistent branching population the progeny of any particle converges to $-\infty$ in a uniform way or dies out. The next proposition says that a similar conclusion applies to the progeny of any family. Let $\varrho_{n}^{(i)}$ be the progeny of the family $T_{W_{i}} \Gamma_{i}$ at time $n$ in the stationary branching population starting with $\Pi_{e_{\lambda}}^{\chi}$.

Proposition 2.8. Assume that $\varphi(\lambda)=0, \lambda>0$, and $\varphi^{\prime}(\lambda)>0$. If $\chi$ is critical or supercritical, then

$$
\mathrm{P}\left[\text { for all } i \in \mathbb{N}: \lim _{n \rightarrow \infty} \max \varrho_{n}^{(i)}=-\infty\right]=1 .
$$

In particular, every family a.s. produces only finitely many maximal particles and only finitely many particles above any fixed level. Also, it follows that there are a.s. infinitely many families (at time 0) which manage to produce a maximal particle at some time in the sequel. Let us stress that the convergence in Proposition 2.8 is not uniform in $i$, since otherwise the whole branching system would drift to $-\infty$ as in the case of local extinction.

Remark 2.3. If the cluster distribution $\chi$ allows only for integer-valued displacements of the particles then it is natural to consider a branching population on $\mathbb{Z}$ starting from a Poisson point process on $\mathbb{Z}$ with intensity $\sum_{u \in \mathbb{Z}} \mathrm{e}^{-\lambda u} \delta_{u}$. Our results have straightforward analogues in this setting. The nonlattice condition in Theorem 2.1 should be replaced by the assumption that $J$ is aperiodic. 


\subsection{The boundary case}

We consider a system of particles starting from a Poisson point process on $\mathbb{R}$ with intensity $\mathrm{e}^{-\lambda u} \mathrm{~d} u$, where $\lambda>0$, and performing independent binary branching Brownian motions with drift $-c$, where $c>0$. This means that each particle performs a standard Brownian motion with drift $-c$; after an exponential time with mean 1 it splits into two particles which behave in the same way as the original particle independently of each other. Branching Brownian motion is a continuous-time process, but its restriction to integer times belongs to the class of processes considered here. It is readily checked that $\varphi(\lambda)=\left(\lambda^{2} / 2\right)-c \lambda+1$. For $c>\sqrt{2}$, the equation $\varphi(\lambda)=0$ has two different solutions, $\lambda_{ \pm}=c \pm \sqrt{c^{2}-2}$. By Theorem 1.1, the measure $e_{\lambda_{+}}$corresponds to a persistent branching population, while the measure $e_{\lambda_{-}}$corresponds to a branching population which becomes locally extinct. If $c=\sqrt{2}$ then there is only one solution, $\lambda=\sqrt{2}$, and we are in the supercritical boundary case. Lalley and Selke [16] conjectured that in this case the branching population should be persistent. We will prove that this is not the case.

Theorem 2.2. Consider a system of independent binary branching Brownian motions with drift $-\sqrt{2}$ starting from the Poisson point process on $\mathbb{R}$ with intensity $e_{\sqrt{2}}$. Denote by $\pi_{n}$ the point processes formed by the particles at time $n$. Then, $\pi_{n}$ converges as $n \rightarrow \infty$ to the empty process.

However, the second invariant measure for the branching population with $c=\sqrt{2}$ still exists in a somewhat more general sense. An easy computation shows that the signed measure $v(\mathrm{~d} u)=u \mathrm{e}^{-\sqrt{2} u} \mathrm{~d} u$ is a solution of the convolution equation (1.3) in the case $c=\sqrt{2}$. It has recently been shown in [2] that the branching population starting from a Poisson point process with intensity $-u \mathrm{e}^{-\sqrt{2} u} \mathbf{1}_{\{u<0\}} \mathrm{d} u$ converges to a nontrivial limiting point process. In this connection, see also [1] and [19].

Let us finally discuss the critical boundary case. Consider a critical branching population without drift. This means that $\varphi(0)=\varphi^{\prime}(0)=0$. In this case, the convolution equation (1.3) has up to a constant multiple just one solution, the Lebesgue measure. To see this, note that we have $K=\{0\}$ in (1.4). It is known that the corresponding branching population suffers local extinction; see [15]. However, it is easy to check that the signed measure $v(\mathrm{~d} u)=u \mathrm{~d} u$ also solves the convolution equation (1.3). We conjecture that a critical branching population without drift starting from a Poisson point process with intensity $u \mathbf{1}_{\{u>0\}} \mathrm{d} u$ converges to a nontrivial limiting point process as the time goes to $\infty$.

\section{Proofs of Theorem 1.1 and Theorem 2.2}

\subsection{Two lemmas on large deviations}

Recall that $I(z)=\sup _{t \in \mathbb{R}}(z t-\varphi(t))$ is the Legendre-Fenchel transform of $\varphi$. We need the following simple Chernoff-type estimate.

Lemma 3.1. Let $\left\{\chi_{n}: n \in \mathbb{N}_{0}\right\}$ be a branching random walk with cluster distribution $\chi$ satisfying (1.2). Then, for every $n \in \mathbb{N}$ and $a \geq n \varphi^{\prime}(0)$,

$$
\mathrm{P}\left[\max \chi_{n} \geq a\right] \leq \mathrm{e}^{-n I(a / n)} .
$$

Proof. Let $J^{[n]}=J * \cdots * J$ be the $n$th convolution power of $J$. Note that $J^{[n]}$ is the intensity of $\chi_{n}$. We have, for every $t \geq 0$,

$$
\mathrm{P}\left[\max \chi_{n} \geq a\right] \leq J^{[n]}([a, \infty)) \leq \int_{\mathbb{R}} \mathrm{e}^{t(u-a)} J^{[n]}(\mathrm{d} u)=\mathrm{e}^{-n(a t / n-\varphi(t))} .
$$


The proof is completed by noting that $\sup _{t \geq 0}(a t / n-\varphi(t))=I(a / n)$, where the supremum can be taken over $t \geq 0$ since $a / n \geq \varphi^{\prime}(0)$.

Lemma 3.2. Let $\left\{\chi_{n}: n \in \mathbb{N}_{0}\right\}$ be a branching random walk with a supercritical cluster distribution $\chi$ satisfying (1.2). If $t \geq 0$ is such that $\varphi^{\prime}(t)<\beta_{0}$ then

$$
\lim _{n \rightarrow \infty} \frac{1}{n} \log \mathrm{E}\left[\mathrm{e}^{t \max \chi_{n}}\right]=\beta_{0} t .
$$

Remark 3.1. If $\varphi^{\prime}(t)>\beta_{0}$ then the limit is equal to $\varphi(t)$; see Proposition 2.3.

Proof of Lemma 3.2. Write $M_{n}=\max \chi_{n}$. By (2.1) we have $\lim _{n \rightarrow \infty} M_{n} / n=\beta_{0}$ a.s. on survival. It follows in a straightforward way that

$$
\liminf _{n \rightarrow \infty} \frac{1}{n} \log \mathrm{E}\left[\mathrm{e}^{t M_{n}}\right] \geq \beta_{0} t .
$$

We prove the converse inequality. Trivially, $\mathrm{E}\left[\mathrm{e}^{t M_{n}} \mathbf{1}_{\left\{M_{n} \leq \beta_{0} n\right\}}\right] \leq \mathrm{e}^{\beta_{0} t n}$. Fix $\varepsilon>0$. Then, by Lemma 3.1,

$$
\mathrm{E}\left[\mathrm{e}^{t M_{n}} \mathbf{1}_{\left\{M_{n}>\beta_{0} n\right\}}\right] \leq \sum_{z \in \beta_{0}+\varepsilon \mathbb{N}_{0}} \mathrm{e}^{t(z+\varepsilon) n} \mathrm{P}\left[M_{n} \geq z n\right] \leq \mathrm{e}^{\varepsilon t n} \sum_{z \in \beta_{0}+\varepsilon \mathbb{N}_{0}} \mathrm{e}^{(t z-I(z)) n}
$$

By convexity and since $I\left(\beta_{0}\right)=0$, we have $I(z) \geq I^{\prime}\left(\beta_{0}\right)\left(z-\beta_{0}\right)$. Note also that $t=$ $I^{\prime}\left(\varphi^{\prime}(t)\right)<I^{\prime}\left(\beta_{0}\right)$. It follows that

$$
\mathrm{E}\left[\mathrm{e}^{t M_{n}} \mathbf{1}_{\left\{M_{n}>\beta_{0} n\right\}}\right] \leq \mathrm{e}^{\varepsilon t n} \mathrm{e}^{\beta_{0} I^{\prime}\left(\beta_{0}\right) n} \sum_{z \in \beta_{0}+\varepsilon \mathbb{N}_{0}} \mathrm{e}^{\left(t-I^{\prime}\left(\beta_{0}\right)\right) z n} \leq C \mathrm{e}^{\left(\beta_{0}+\varepsilon\right) t n} .
$$

Bringing everything together, we obtain the statement of the lemma.

\subsection{Kallenberg's stability criterion}

Our proof of the persistence part of Theorem 1.1 is based on the backward tree construction introduced by Kallenberg [15] in the context of a critical branching population on $\mathbb{R}^{d}$ starting with a homogeneous Poisson point process. This approach has been generalized in [17, Section 2.4] (see also [23]) to arbitrary branching populations on Polish spaces. The backward tree construction appeared in many different contexts; see the references in [18].

Recall that we consider a branching population on $\mathbb{R}^{d}$ with spatially homogeneous cluster distribution $\chi$ having intensity $J$. We assume that $\lambda \in \mathbb{R}^{d}$ is such that $\varphi(\lambda)=0$. Let $o$ be an individual which is alive at time 0 and is located at the origin in $\mathbb{R}^{d}$. The idea of Kallenberg's method is to trace back the history of individual $o$. First, we define the set of the direct ancestors of $o$. Let $D$ be a probability measure on $\mathbb{R}^{d}$ defined by

$$
D(B)=\int_{B} \mathrm{e}^{\langle\lambda, u\rangle} J(\mathrm{~d} u),
$$

where $B \subset \mathbb{R}^{d}$ is Borel. Since $\varphi(\lambda)=0, D$ is indeed a probability measure. Let $\left\{\xi_{n}: n \in \mathbb{N}\right\}$ be independent, $\mathbb{R}^{d}$-valued random variables with distribution $D$. Let $S_{n}=\xi_{1}+\cdots+\xi_{n}$, $S_{0}=0$, be the corresponding random walk. It is convenient to think of $-S_{n}$ as the position 
of the direct ancestor of $o$ which is alive at time $-n$. So, $-S_{1}$ is the position of the father of $o$ alive at time $-1,-S_{2}$ is the position of the grandfather of $o$ alive at time -2 , etc. Now we define the set of relatives of $o$ alive at time 0 , i.e. the set of its brothers, cousins, etc. Let $\left\{\chi_{(z)}: z \in \mathbb{R}^{d}\right\}$ be the system of Palm measures of the point process $\chi$; see [17, Section 1.8] and [21, Chapters 8 and 9]. Denote by $\left\{\chi_{(z)}^{!}: z \in \mathbb{R}^{d}\right\}$ the reduced Palm measures, where $\chi_{(z)}^{!}$ is obtained from $\chi_{(z)}$ by removing the point $z$. Conditioned on $\xi_{1}, \xi_{2}, \ldots$, let $\kappa_{n}=\sum_{k=1}^{k_{n}} \delta_{z_{k: n}}$, $n \in \mathbb{N}$, be independent point processes such that $\kappa_{n}$ has the same law as $\chi_{\left(\xi_{n}\right)}^{!}$. Think of $-S_{n}+z_{k: n}, k=1, \ldots, k_{n}$, as the locations of the brothers (alive at time $-(n-1)$ ) of the ancestor of $o$ located at $-S_{n-1}$. Also, let $\left\{\chi_{l}^{(k, n)}: l \in \mathbb{N}_{0}\right\}$, where $n \in \mathbb{N}$ and $k=1, \ldots, k_{n}$, be independent copies of the original branching random walk $\left\{\chi_{l}: l \in \mathbb{N}_{0}\right\}$ generated by the cluster distribution $\chi$. Think of $\chi_{l}^{(k, n)}$ as the point process describing the displacements of the offspring in the $l$ th generation of the individual located at time $-(n-1)$ at $-S_{n}+z_{k: n}$. Then, we define a point process $\rho_{n}$ by

$$
\rho_{n}=\sum_{k=1}^{k_{n}} T_{-S_{n}+z_{k: n}}\left(\chi_{n-1}^{(k, n)}\right) .
$$

Think of $\rho_{n}$ as the point process describing the positions of the relatives of $o$ which are alive at time 0 and have the same ancestor at time $-n$ as $o$. Let $\rho_{0}=\delta_{0}$. The next theorem is a specification of [17, Proposition 2.4.3] to our setting.

Theorem 3.1. (Kallenberg's stability criterion.) The branching population starting from a Poisson point process with intensity $e_{\lambda}$ is persistent if and only if the measure $\rho:=\sum_{n=0}^{\infty} \rho_{n}$ is locally finite with probability 1 .

\subsection{Proof of part 1 of Theorem 1.1}

First we introduce some notation which is needed to handle the case $d \geq 2$, but may be ignored in the case $d=1$. Without loss of generality, we may assume that $\lambda=(l, 0, \ldots, 0)$ for some $l>0$; otherwise, we can rotate the coordinates. We denote by $P: \mathbb{R}^{d} \rightarrow \mathbb{R}$ the projection operator $P\left(l_{1}, \ldots, l_{d}\right)=l_{1}$. We may project the cluster distribution $\chi$ to $\mathbb{R}$ via $P$. The resulting cluster distribution $\chi_{P}$ has intensity $J_{P}(B)=J\left(P^{-1} B\right)$, where $B \subset \mathbb{R}$ is Borel, and the log-Laplace transform of $J_{P}$ is given by $\varphi_{P}(t)=\varphi(t, 0, \ldots, 0), t \in \mathbb{R}$. Let $I_{P}(z)=\sup _{t \in \mathbb{R}}\left(z t-\varphi_{P}(t)\right)$ be the Legendre-Fenchel transform of $\varphi_{P}$. We have $\varphi_{P}(l)=0$. Also, the assumption that $\langle\lambda, \nabla \varphi(\lambda)\rangle>0$ implies that $\varphi_{P}^{\prime}(l)>0$.

By Theorem 3.1, it suffices to verify that $\rho(A)$ is finite a.s. for $A$ being a half-space set of the form $[a, \infty) \times \mathbb{R}^{d-1}, a \in \mathbb{R}$. Denote by $\mathcal{A}_{\xi, \kappa}$ the $\sigma$-algebra generated by the random variables $\left\{\xi_{n}: n \in \mathbb{N}\right\}$ and the point processes $\left\{\kappa_{n}: n \in \mathbb{N}\right\}$. We have, by (3.2), Lemma 3.1, and the convexity of the function $I_{P}$,

$$
\begin{aligned}
& \mathrm{P}\left[\rho_{n}(A)>0 \mid \mathcal{A}_{\xi, k}\right] \\
& \quad \leq \sum_{k=1}^{k_{n}} \mathrm{P}\left[\max P\left(\chi_{n-1}^{(k, n)}\right) \geq P\left(S_{n}\right)-P\left(z_{k: n}\right)+a\right] \\
& \quad \leq \sum_{k=1}^{k_{n}} \exp \left\{-(n-1) I_{P}\left(\frac{P\left(S_{n}\right)-P\left(z_{k: n}\right)+a}{n-1}\right)\right\} \\
& \quad \leq \sum_{k=1}^{k_{n}} \exp \left\{-(n-1) I_{P}\left(\frac{P\left(S_{n}\right)}{n-1}\right)+I_{P}^{\prime}\left(\frac{P\left(S_{n}\right)}{n-1}\right)\left(P\left(z_{k: n}\right)-a\right)\right\} .
\end{aligned}
$$


The expectation of $\xi_{1}$ is given by $\mathrm{E}\left[\xi_{1}\right]=\int_{\mathbb{R}^{d}} u D(\mathrm{~d} u)=\nabla \varphi(\lambda)$. By the law of large numbers,

$$
\frac{P\left(S_{n}\right)}{n} \rightarrow P(\nabla \varphi(\lambda))=\varphi_{P}^{\prime}(l)>0 \quad \text { a.s. }
$$

By the properties of the Legendre-Fenchel transform we have $I_{P}\left(\varphi_{P}^{\prime}(l)\right)=l \varphi_{P}^{\prime}(l)$ and $I_{P}^{\prime}\left(\varphi_{P}^{\prime}(l)\right)=l$. Take $\varepsilon>0$. It follows that, with probability 1 , we have, for sufficiently large $n$,

$$
I_{P}\left(\frac{P\left(S_{n}\right)}{n-1}\right)>l \varphi_{P}^{\prime}(l)-\varepsilon, \quad l-\varepsilon<I_{P}^{\prime}\left(\frac{P\left(S_{n}\right)}{n-1}\right)<l+\varepsilon .
$$

Thus, we have

$$
\mathrm{P}\left[\rho_{n}(A)>0 \mid \mathcal{A}_{\xi, \kappa}\right] \leq C \mathrm{e}^{-\left(l \varphi_{P}^{\prime}(l)-\varepsilon\right) n} Y_{n},
$$

where $\left\{Y_{n}: n \in \mathbb{N}\right\}$ are random variables defined by

$$
Y_{n}=\sum_{k=1}^{k_{n}}\left(\mathrm{e}^{(l+\varepsilon) P\left(z_{k: n}\right)}+\mathrm{e}^{(l-\varepsilon) P\left(z_{k: n}\right)}\right)=\int_{\mathbb{R}^{d}}\left(\mathrm{e}^{\left\langle\lambda_{+\varepsilon}, u\right\rangle}+\mathrm{e}^{\left\langle\lambda_{-\varepsilon}, u\right\rangle}\right) \kappa_{n}(\mathrm{~d} u)
$$

and $\lambda_{ \pm \varepsilon}=(l \pm \varepsilon, 0, \ldots, 0)$. We will show that $\mathrm{E}\left[Y_{n}\right]$ is finite. Define a function $f: \mathbb{M} \rightarrow$ $[0,+\infty]$ (where $\mathbb{M}$ is the space of all locally finite counting measures on $\mathbb{R}^{d}$ ) by $f(\beta)=$ $\int_{\mathbb{R}^{d}}\left(\mathrm{e}^{\left\langle\lambda_{+\varepsilon}, u\right\rangle}+\mathrm{e}^{\left\langle\lambda_{-\varepsilon}, u\right\rangle}\right) \beta(\mathrm{d} u)$. Recalling the definition of $\kappa_{n}$ we have

$$
\begin{aligned}
\mathrm{E}\left[Y_{n}\right] & =\int_{\mathbb{R}^{d}} \mathrm{E}\left[f\left(\chi_{(u)}^{!}\right)\right] \mathrm{e}^{\langle\lambda, u\rangle} J(\mathrm{~d} u) \\
& \leq \int_{\mathbb{R}^{d}} \mathrm{E}\left[f\left(\chi_{(u)}\right)\right] \mathrm{e}^{\langle\lambda, u\rangle} J(\mathrm{~d} u) \\
& =\int_{\mathbb{R}^{d}} \mathrm{E}\left[h\left(u, \chi_{(u)}\right)\right] J(\mathrm{~d} u),
\end{aligned}
$$

where $h: \mathbb{R}^{d} \times \mathbb{M} \rightarrow[0,+\infty]$ is defined by $h(u, \beta)=\mathrm{e}^{\langle\lambda, u\rangle} f(\beta)$. By the definition of the Palm measures we have

$$
\int_{\mathbb{R}^{d}} \mathrm{E}[h(u, \chi(u))] J(\mathrm{~d} u)=\mathrm{E}\left[\int_{\mathbb{R}^{d}} h(u, \chi) \chi(\mathrm{d} u)\right] .
$$

Using this, we obtain

$$
\begin{aligned}
\mathrm{E}\left[Y_{n}\right] & \leq \mathrm{E}\left[\int_{\mathbb{R}^{d}} h(u, \chi) \chi(\mathrm{d} u)\right] \\
& =\mathrm{E}\left[\left(\int_{\mathbb{R}^{d}}\left(\mathrm{e}^{\left\langle\lambda_{+\varepsilon}, u\right\rangle}+\mathrm{e}^{\left\langle\lambda_{-\varepsilon}, u\right\rangle}\right) \chi(\mathrm{d} u)\right)\left(\int_{\mathbb{R}^{d}} \mathrm{e}^{\langle\lambda, u\rangle} \chi(\mathrm{d} u)\right)\right] .
\end{aligned}
$$

Using (1.1), we will show that the right-hand side is finite. Write $\chi=\sum_{k=1}^{L} \delta_{u_{k}}$, where $L=\chi\left(\mathbb{R}^{d}\right)$ and the $u_{k}, k=1, \ldots, L$, are (in general, dependent) random variables. Then, for 
every $\lambda_{1}, \lambda_{2} \in \mathbb{R}^{d}$

$$
\begin{aligned}
\mathrm{E}\left[\left(\int_{\mathbb{R}^{d}} \mathrm{e}^{\left\langle\lambda_{1}, u\right\rangle} \chi(\mathrm{d} u)\right)\left(\int_{\mathbb{R}^{d}} \mathrm{e}^{\left\langle\lambda_{2}, u\right\rangle} \chi(\mathrm{d} u)\right)\right] & =\mathrm{E}\left[\sum_{k_{1}=1}^{L} \sum_{k_{2}=1}^{L} \mathrm{e}^{\left\langle\lambda_{1}, u_{k_{1}}\right\rangle} \mathrm{e}^{\left\langle\lambda_{2}, u_{k_{2}}\right\rangle}\right] \\
& \leq \frac{1}{2} \mathrm{E}\left[\sum_{k_{1}=1}^{L} \sum_{k_{2}=1}^{L}\left(\mathrm{e}^{\left\langle 2 \lambda_{1}, u_{k_{1}}\right\rangle}+\mathrm{e}^{\left\langle 2 \lambda_{2}, u_{k_{2}}\right\rangle}\right)\right] \\
& =\frac{1}{2} \mathrm{E}\left[L\left(\sum_{k=1}^{L}\left(\mathrm{e}^{\left\langle 2 \lambda_{1}, u_{k}\right\rangle}+\mathrm{e}^{\left\langle 2 \lambda_{2}, u_{k}\right\rangle}\right)\right)\right]
\end{aligned}
$$

The right-hand side is finite by $(1.1)$ and, hence, $\mathrm{E}\left[Y_{n}\right]<\infty$. Recall that in part 1 of Theorem 1.1 we assume that $l \varphi_{P}^{\prime}(l)>0$. It follows that

$$
\sum_{n=0}^{\infty} \mathrm{e}^{-\left(l \varphi_{P}^{\prime}(l)-\varepsilon\right) n} Y_{n}<\infty \quad \text { a.s. }
$$

It follows from (3.3) that, for almost every (a.e.) choice of $\left\{\xi_{i}, \kappa_{i}: i \in \mathbb{N}\right\}$, we have

$$
\sum_{n=0}^{\infty} \mathrm{P}\left[\rho_{n}(A)>0 \mid\left\{\xi_{i}, \kappa_{i}: i \in \mathbb{N}\right\}\right]<\infty
$$

By the Borel-Cantelli lemma, for a.e. $\left\{\xi_{i}, \kappa_{i}: i \in \mathbb{N}\right\}, \rho(A)=\sum_{n=0}^{\infty} \rho_{n}(A)$ is finite a.s. Thus, $\rho(A)$ is finite a.s. Theorem 3.1 completes the proof.

\subsection{Proof of part 2 of Theorem 1.1}

Proof of Proposition 2.2. Recall the definition of $\pi_{n}$ in (2.2). The point process

$$
\pi_{n}^{*}:=\sum_{i=1}^{\infty} \delta_{U_{i}+\max } \chi_{n}^{(i)}
$$

is obtained from the Poisson point process $\pi_{0}=\sum_{i=1}^{\infty} \delta_{U_{i}}$ by independent shifting. Hence, $\pi_{n}^{*}$ is itself a Poisson point process on $\mathbb{R}$ with intensity $\mathrm{E}\left[\mathrm{e}^{\lambda \max \chi_{n}}\right] \mathrm{e}^{-\lambda u} \mathrm{~d} u$. For every $\beta>\beta_{0}$, we have

$$
\sum_{n=1}^{\infty} \mathrm{P}\left[\max \pi_{n}>\beta n\right] \leq \sum_{n=1}^{\infty} \mathrm{E}\left[\pi_{n}^{*}((\beta n, \infty))\right]=\frac{1}{\lambda} \sum_{n=1}^{\infty}\left(\mathrm{e}^{-\lambda \beta n} \mathrm{E}\left[\mathrm{e}^{\lambda \max \chi_{n}}\right]\right)<\infty,
$$

where the last step follows from Lemma 3.2 (note that $\varphi^{\prime}(\lambda)<\beta_{0}$ ). By the Borel-Cantelli lemma, we have $\lim \sup _{n \rightarrow \infty}(1 / n) \max \pi_{n} \leq \beta_{0}$ a.s. The converse inequality follows immediately from (2.1).

Proof of part 2 of Theorem 1.1. First let $d=1$. For concreteness, assume that $\lambda>0$. Then, $\varphi^{\prime}(0)<0, \beta_{0}<0$, and Proposition 2.2 implies that $\lim _{n \rightarrow \infty} \max \pi_{n}=-\infty$ a.s. It follows that $\pi_{n}$ converges weakly to the empty process.

We consider the case $d \geq 2$. Without restriction of generality, let $\lambda=(l, 0, \ldots, 0)$ with $l>0$. Let $A \subset \mathbb{R}^{d}$ be a bounded Borel set. We will show that, with probability $1, \pi_{n}(A)=0$ for sufficiently large $n$. Let $E_{n}=\mathbb{R} \times\left[-n^{2}, n^{2}\right]^{d-1}$ and $\bar{E}_{n}=\mathbb{R}^{d} \backslash E_{n}$. Let $N_{n}$ and $\bar{N}_{n}$ be random 
variables counting the numbers of particles of the point process $\pi_{n}$ which are in the set $A$ and whose ancestor at time 0 was in the set $E_{n}$, or, respectively, $\bar{E}_{n}$. Clearly, $\pi_{n}(A)=N_{n}+\bar{N}_{n}$. After elementary transformations we obtain

$$
\mathrm{E}\left[\bar{N}_{n}\right]=\int_{\bar{E}_{n}} \mathrm{e}^{-\langle\lambda, u\rangle} J^{[n]}(A-u) \mathrm{d} u=\int_{A} \mathrm{e}^{-\langle\lambda, a\rangle} D^{[n]}\left(a-\bar{E}_{n}\right) \mathrm{d} a,
$$

where $D^{[n]}=D * \cdots * D$ is the $n$th convolution power of the probability measure $D$ defined in (3.1). By the Cramér-Chernoff bound, there is a $c>0$ such that $D^{[n]}\left(a-\bar{E}_{n}\right)=O\left(\mathrm{e}^{-c n}\right)$ uniformly in $a \in A$. Hence, $\mathrm{P}\left[\bar{N}_{n}>0\right] \leq \mathrm{E}\left[\bar{N}_{n}\right]=O\left(\mathrm{e}^{-c n}\right)$. By the Borel-Cantelli lemma, $\bar{N}_{n}=0$ for all but finitely many $n$ a.s.

Let us show that, with probability $1, N_{n}=0$ for all but finitely many $n$. Consider the progeny of all particles which start at time 0 in the cylinder set $\mathbb{R} \times[0,1]^{d-1}$. Let us project these particles onto the first coordinate $\mathbb{R}$ via the projection operator $P$; see Section 3.3. We obtain a branching population $\left\{\pi_{n}^{(P)}: n \in \mathbb{N}_{0}\right\}$ on $\mathbb{R}$ starting from a Poisson point process with intensity $\mathrm{e}^{-l x}$. By Proposition 2.2, (see also (3.6)), there are $\beta<0$ and $c>0$ such that $\mathrm{P}\left[\max \pi_{n}^{(P)}>\beta n\right]=O\left(\mathrm{e}^{-c n}\right)$. Divide the set $E_{n}$ into $\left(2 n^{2}\right)^{d-1}$ sets which are translates of the set $\mathbb{R} \times[0,1]^{d-1}$ and apply to each of them the above considerations. Let $R_{n}$ be the largest among the first coordinates of the offspring in the $n$th generation of the particles which started in $E_{n}$. It follows that $\mathrm{P}\left[R_{n}>\beta n\right]=O\left(n^{2 d-2} \mathrm{e}^{-c n}\right)=O\left(\mathrm{e}^{-c n / 2}\right)$. By the Borel-Cantelli lemma, $R_{n} \rightarrow-\infty$ a.s. It follows that, with probability $1, N_{n}=0$ for all but finitely many $n$. This completes the proof.

\subsection{Proof of Theorem 2.2}

Let $\chi_{n}$ be the point process formed by the particles at time $n$ in a single binary branching Brownian motion with drift $-\sqrt{2}$. Write $M_{n}=\max \chi_{n}$. It suffices to show that

$$
\lim _{n \rightarrow \infty} \mathrm{E}\left[\mathrm{e}^{\sqrt{2} M_{n}}\right]=0
$$

Fix $\varepsilon>0$. Note that the expected number of particles at time $n$ in the binary branching Brownian motion is $\mathrm{e}^{n}$. Denoting by $\bar{\Phi}$ the tail of the standard normal distribution function we have a trivial estimate

$$
\mathrm{P}\left[M_{n} \geq y\right] \leq \mathrm{E}\left[\chi_{n}([y, \infty))\right]=\mathrm{e}^{n} \bar{\Phi}\left(\frac{y+\sqrt{2} n}{\sqrt{n}}\right) \leq \frac{C}{\sqrt{n} \mathrm{e}^{\sqrt{2} y}} \mathrm{e}^{-y^{2} / 2 n} .
$$

It follows that, for a sufficiently large $c>0$,

$$
\begin{aligned}
\mathrm{E}\left[\mathrm{e}^{\sqrt{2} M_{n}} \mathbf{1}_{\left\{M_{n} \geq[c \sqrt{n}]\right\}}\right] & \leq C \sum_{k=[c \sqrt{n}]}^{\infty} \mathrm{e}^{\sqrt{2} k} \mathrm{P}\left[M_{n} \geq k\right] \\
& \leq \frac{C}{\sqrt{n}} \sum_{k=[c \sqrt{n}]}^{\infty} \mathrm{e}^{-k^{2} / 2 n} \\
& \leq C \int_{c-2 / \sqrt{n}}^{\infty} \mathrm{e}^{-s^{2} / 2} \mathrm{~d} s \\
& \leq \varepsilon .
\end{aligned}
$$


Write $l_{n}=3 \log n / 2 \sqrt{2}$. By Proposition 3 of [6], there is a $B=B(c)$ such that, for all $-l_{n} \leq y \leq c \sqrt{n}$, we have the following estimate:

$$
\mathrm{P}\left[M_{n} \geq y\right] \leq \frac{B}{n^{3 / 2} \mathrm{e}^{\sqrt{2} y}} .
$$

This is stated in [6] for $c=1$ only, but the proof is valid for every $c>0$. It follows that

$$
\mathrm{E}\left[\mathrm{e}^{\sqrt{2} M_{n}} \mathbf{1}_{\left\{-\left[l_{n}\right] \leq M_{n} \leq[c \sqrt{n}]\right\}}\right] \leq \sum_{k=-\left[l_{n}\right]}^{[c \sqrt{n}]} \mathrm{e}^{\sqrt{2}(k+1)} \mathrm{P}\left[M_{n} \geq k\right]=O\left(\frac{1}{n}\right)
$$

Trivially, we have $\lim _{n \rightarrow \infty} \mathrm{E}\left[\mathrm{e}^{\sqrt{2} M_{n}} \mathbf{1}_{\left\{M_{n} \leq-l_{n}\right\}}\right]=0$. Bringing everything together, we obtain (3.7).

\section{Proofs of Propositions 2.1, 2.3, 2.4, 2.7, and 2.8}

\subsection{Proofs of Propositions 2.3 and 2.4}

Assume first that $\varphi(t)=0$ and $\varphi^{\prime}(t)>\beta_{0}$, which, in fact, implies that $\varphi^{\prime}(t)>0$. Then, the branching population $\left\{\pi_{n}: n \in \mathbb{N}_{0}\right\}$ starting from a Poisson point process with intensity $e_{t}$ is persistent by Theorem 1.1. That is, $\pi_{n}$ converges weakly, as a random element of $\mathbb{M}$, to some limiting point process $\Pi_{e_{t}}^{\chi}$ with intensity $e_{t}$. We will argue that this implies that the random variable $\max \pi_{n}$ converges to $\max \Pi_{e_{t}}^{\chi}$ weakly. Let $\mathbb{M}^{\prime}$ be the space of locally finite counting measures on $(-\infty,+\infty$ ] endowed with the vague topology. In fact, the convergence of $\pi_{n}$ to $\Pi_{e_{t}}^{\chi}$ holds also on the space $\mathbb{M}^{\prime}$, the proof being the same as in Theorem 1.1. Define a function $f: \mathbb{M}^{\prime} \rightarrow \mathbb{R}$ by $f(\beta)=\max \beta$ if $\max \beta$ is finite, and $f(\beta)=0$ otherwise. Then, $f$ is continuous on the set $\left\{\beta \in \mathbb{M}^{\prime}: \max \beta<\infty\right\}$. This set has full measure with respect to the law of $\Pi_{e_{t}}^{\chi}$. Indeed, max $\Pi_{e_{t}}^{\chi}<\infty$ a.s. since $e_{t}$, the intensity of $\Pi_{e_{t}}^{\chi}$, is integrable over $(0,+\infty)$. By the continuous mapping theorem, it follows that max $\pi_{n}$ converges to max $\Pi_{e_{t}}^{\chi}$ weakly.

On the other hand, we have $\max \pi_{n}=\max \pi_{n}^{*}$, where $\pi_{n}^{*}=\sum_{i=1}^{\infty} \delta_{U_{i}+\max \chi_{n}^{(i)}}$ and the same notation as in (2.2) is used. Since $\pi_{n}^{*}$ is obtained from the Poisson point process $\pi_{0}=\sum_{i=1}^{\infty} \delta_{U_{i}}$ by independent shifting, $\pi_{n}^{*}$ is itself a Poisson point process with intensity $\mathrm{E}\left[\mathrm{e}^{t \max x_{n}}\right] \mathrm{e}^{-t u} \mathrm{~d} u$. It follows that

$$
\mathrm{P}\left[\max \pi_{n} \leq z\right]=\mathrm{P}\left[\pi_{n}^{*}((z,+\infty))=0\right]=\exp \left\{-\frac{1}{t} \mathrm{E}\left[\mathrm{e}^{t \max \chi_{n}}\right] \mathrm{e}^{-t z}\right\} .
$$

The weak convergence of $\max \pi_{n}$ to $\max \Pi_{e_{t}}^{\chi}$ implies that $\mathrm{P}\left[\max \pi_{n} \leq z\right]$ must have a limit in $(0,1)$ for at least one $z \in \mathbb{R}$. Hence, $c(t)=\lim _{n \rightarrow \infty} \mathrm{E}\left[\mathrm{e}^{t \max \chi_{n}}\right]$ exists in $(0, \infty)$.

If $\varphi(t) \neq 0$ and $\varphi^{\prime}(t)>\beta_{0}$, we consider another branching random walk $\left\{\tilde{\chi}_{n}: n \in \mathbb{N}_{0}\right\}$ obtained by adding to $\left\{\chi_{n}: n \in \mathbb{N}_{0}\right\}$ a drift $-\varphi(t) n / t$, that is, $\tilde{\chi}_{n}=T_{-\varphi(t) n / t}\left(\chi_{n}\right)$. The logLaplace transform $\tilde{\varphi}$ of $\tilde{\chi}_{1}$ satisfies $\tilde{\varphi}(t)=0$ and $\tilde{\varphi}^{\prime}(t)>\beta_{0}-\varphi(t) / t=\tilde{\beta}_{0}$, where $\tilde{\beta}_{0}$ is the analogue of $\beta_{0}$ for the function $\tilde{I}(z)=\sup _{s \in \mathbb{R}}(s z-\tilde{\varphi}(s))$. By the above,

$$
\lim _{n \rightarrow \infty} \mathrm{e}^{-\varphi(t) n} \mathrm{E}\left[\mathrm{e}^{t \max \chi_{n}}\right]=\lim _{n \rightarrow \infty} \mathrm{E}\left[\mathrm{e}^{t \max \tilde{\chi}_{n}}\right] \in(0, \infty) .
$$

This completes the proof of the first part of Proposition 2.3. The second part follows from Lemma 3.2. Proposition 2.4 follows by taking the limit $n \rightarrow \infty$ in (4.1). 


\subsection{Proof of Proposition 2.1}

The idea of the proof is as follows. At time 0 , the density of particles at $-u n$ is $\mathrm{e}^{\lambda u n}$, and the probability that a particle at $-u n$ will produce at time $n$ an offspring, say, on the positive half-axis is approximately $\mathrm{e}^{-n I(u)}$; cf. Lemma 3.1. Thus, the ancestor at time 0 of the particle which is maximal at time $n$ is most likely to be found at position approximately $-u n$, where $u$ is such that $\lambda u-I(u)$ is maximal. The maximizer is given by $u=\varphi^{\prime}(\lambda)$, whence the statement.

Let us provide a rigorous argument justifying this. Fix some $\varepsilon>0$, and write $\alpha_{ \pm}(\varepsilon)=$ $\varphi^{\prime}(\lambda) \pm \varepsilon$. We estimate the probability $P_{n}^{+}(\varepsilon)$ that some offspring of some particle which started at time 0 below the level $-\alpha_{+}(\varepsilon) n$ will be above the level $-\sqrt{n}$ at time $n$ :

$$
\begin{aligned}
P_{n}^{+}(\varepsilon) & :=\mathrm{P}\left[\max _{i \in \mathbb{N}, U_{i}<-\alpha_{+}(\varepsilon) n}\left(U_{i}+\max \chi_{n}^{(i)}\right) \geq-\sqrt{n}\right] \\
& \leq \int_{\alpha_{+}(\varepsilon) n}^{\infty} \mathrm{e}^{\lambda u} \mathrm{P}\left[\max \chi_{n} \geq u-\sqrt{n}\right] \mathrm{d} u \\
& =n \mathrm{e}^{\lambda \sqrt{n}} \int_{\alpha_{+}(\varepsilon)-1 / \sqrt{n}}^{\infty} \mathrm{e}^{\lambda n v} \mathrm{P}\left[\max \chi_{n} \geq n v\right] \mathrm{d} v \\
& \leq n \mathrm{e}^{\lambda \sqrt{n}} \int_{\alpha_{+}(\varepsilon / 2)}^{\infty} \mathrm{e}^{(\lambda v-I(v)) n} \mathrm{~d} v .
\end{aligned}
$$

Here we have used the substitution $u=n v+\sqrt{n}$ and Lemma 3.1. The last inequality holds if $n$ is sufficiently large. The function $g(v):=\lambda v-I(v)$ is concave, and we have $g\left(\varphi^{\prime}(\lambda)\right)=0$ and $g^{\prime}\left(\varphi^{\prime}(\lambda)\right)=0$. The global maximum of $g$ is attained at $v=\varphi^{\prime}(\lambda)$. By concavity, there are $c_{1}>0$ and $c_{2}>0$ such that $g(v) \leq-c_{1}-c_{2}\left(v-\alpha_{+}(\varepsilon / 2)\right)$ for all $v \geq \alpha_{+}(\varepsilon / 2)$. Hence,

$$
P_{n}^{+}(\varepsilon) \leq n \mathrm{e}^{\lambda \sqrt{n}} \mathrm{e}^{-c_{1} n} \int_{\alpha_{+}(\varepsilon / 2)}^{\infty} \mathrm{e}^{-c_{2}\left(v-\alpha_{+}(\varepsilon / 2)\right) n} \mathrm{~d} v=O\left(\mathrm{e}^{-c_{1} n / 2}\right) .
$$

Analogous considerations apply to $P_{n}^{-}(\varepsilon)$, the probability that some offspring of some particle which started at time 0 above the level $-\alpha_{-}(\varepsilon) n$ will be above the level $-\sqrt{n}$ at time $n$. Hence, $\sum_{n=1}^{\infty} P_{n}^{+}(\varepsilon)<\infty$ and $\sum_{n=1}^{\infty} P_{n}^{-}(\varepsilon)<\infty$. By the Borel-Cantelli lemma, only finitely often a particle starting not in the interval $\left(-\alpha_{+}(\varepsilon) n,-\alpha_{-}(\varepsilon) n\right)$ will be able to produce a particle above the level $-\sqrt{n}$ at time $n$. Also, we have

$$
\sum_{n=1}^{\infty} \mathrm{P}\left[\max \pi_{n}<-\sqrt{n}\right]=\sum_{n=1}^{\infty} \exp \left\{-\frac{1}{\lambda} \mathrm{E}\left[\mathrm{e}^{\lambda \max x_{n}}\right] \mathrm{e}^{\lambda \sqrt{n}}\right\}<\infty,
$$

where the last inequality holds since $\mathrm{E}\left[\mathrm{e}^{\lambda \max \chi_{n}}\right]$ remains bounded by Proposition 2.3 . Hence, only finitely often a maximal particle will be below the level $-\sqrt{n}$. The statement of the proposition follows.

\subsection{Proofs of Propositions 2.7 and 2.8}

We will use the notation introduced in Section 3.2.

Lemma 4.1. If the cluster distribution $\chi$ is subcritical or $\chi(\mathbb{R})=1$ a.s., then the measure $\rho$ introduced in Theorem 3.1 satisfies $\rho(\mathbb{R})<\infty$ a.s. Otherwise, $\rho(\mathbb{R})=\infty$ a.s.

Proof. Let us first show that $k_{n}=\kappa_{n}(\mathbb{R})$ has finite expectation. We have

$$
\mathrm{E}\left[\kappa_{n}(\mathbb{R})\right]=\int_{\mathbb{R}} \mathrm{E}\left[\chi_{(u)}^{!}(\mathbb{R})\right] \mathrm{e}^{\lambda u} J(\mathrm{~d} u) \leq \int_{\mathbb{R}} \mathrm{E}\left[\chi_{(u)}(\mathbb{R})\right] \mathrm{e}^{\lambda u} J(\mathrm{~d} u)=\int_{\mathbb{R}} \mathrm{E}\left[h\left(u, \chi_{(u)}\right)\right] J(\mathrm{~d} u),
$$


where $h: \mathbb{R} \times \mathbb{M} \rightarrow[0,+\infty]$ is a function defined by $h(u, \beta)=\mathrm{e}^{\lambda u} \beta(\mathbb{R})$. By (3.4),

$$
\mathrm{E}\left[\kappa_{n}(\mathbb{R})\right] \leq \mathrm{E}\left[\int_{\mathbb{R}} h(u, \chi) \chi(\mathrm{d} u)\right]=\mathrm{E}\left[\chi(\mathbb{R}) \int_{\mathbb{R}} \mathrm{e}^{\lambda u} \chi(\mathrm{d} u)\right],
$$

which is finite by (1.1). So, $\mathrm{E}\left[k_{n}\right]<\infty$.

In the subcritical case we have $\mathrm{E}[\chi(\mathbb{R})]<1$ and, hence,

$$
\mathrm{E}[\rho(\mathbb{R})]=\sum_{n=0}^{\infty} \mathrm{E}\left[\rho_{n}(\mathbb{R})\right]=1+\sum_{n=1}^{\infty} \mathrm{E}\left[k_{n}\right] \mathrm{E}\left[\chi_{n-1}(\mathbb{R})\right]=1+C \sum_{n=1}^{\infty}(\mathrm{E}[\chi(\mathbb{R})])^{n-1}<\infty .
$$

It follows that $\rho(\mathbb{R})<\infty$ a.s. In the nonsubcritical case we write

$$
\mathrm{P}\left[\rho_{n}(\mathbb{R})>0\right] \geq \mathrm{P}\left[k_{n} \geq 1, \chi_{n-1}^{(1, n)}(\mathbb{R})>0\right]=\mathrm{P}\left[k_{n} \geq 1\right] \mathrm{P}\left[\chi_{n-1}(\mathbb{R})>0\right] .
$$

If we assume that $\mathrm{P}[\chi(\mathbb{R})=1]<1$ then $\mathrm{P}\left[k_{n} \geq 1\right]>0$ is a constant independent of $n$. Furthermore, in the critical case we can find a $c>0$ such that, for all $n \in \mathbb{N}, \mathrm{P}\left[\chi_{n-1}(\mathbb{R})>\right.$ $0]>c / n$; see [3, p. 19]. Here we need the finiteness of $\mathrm{E}\left[\chi(\mathbb{R})^{2}\right]$, which follows from (1.1). In the supercritical case, we even have $\mathrm{P}\left[\chi_{n-1}(\mathbb{R})>0\right]>c$. By the Borel-Cantelli lemma, $\rho(\mathbb{R})=\infty$ a.s.

Lemma 4.2. Let $A \subset \mathbb{M}$ be a Borel set such that if $\beta \in A$ then $T_{u} \beta \in A$ for every $u \in \mathbb{R}$. Let $\varphi(\lambda)=0, \lambda>0$, and $\varphi^{\prime}(\lambda)>0$. Then $\mathrm{P}[\Gamma \in A]=0$ if and only if $\mathrm{P}[\rho \in A]=0$.

Proof. As already observed in Section 2.3, the point process $\Pi_{e_{\lambda}}^{\chi}$ is infinitely divisible. Denote by $\Psi$ its canonical (Lévy) measure; see [21, Chapter 2]. Note that $\Psi$ is a measure on $\mathbb{M}$ which may be infinite in general. On the one hand, the cluster representation of $\Pi_{e_{\lambda}}^{\chi}$ given in Proposition 2.6 implies that we have

$$
\Psi(A)=c \int_{\mathbb{R}} \mathrm{P}\left[T_{u} \Gamma \in A\right] \mathrm{e}^{-\lambda u} \mathrm{~d} u .
$$

Thus, $\mathrm{P}[\Gamma \in A]=0$ if and only if $\Psi(A)=0$. On the other hand, by Theorem 2.4.4 of [17] we have

$$
\Psi_{(u)}(A)=\mathrm{P}\left[T_{u} \rho \in A\right], \quad u \in \mathbb{R},
$$

where $\left\{\Psi_{(u)}: u \in \mathbb{R}\right\}$ is the family of Palm measures of $\Psi$. Thus, $\mathrm{P}[\rho \in A]=0$ if and only if $\Psi_{(u)}(A)=0$ for all $u \in \mathbb{R}$. Finally, by the definition of the Palm measures we have, for every Borel set $B \subset \mathbb{R}$,

$$
\int_{A} \beta(B) \Psi(\mathrm{d} \beta)=\int_{B} \Psi_{(u)}(A) e_{\lambda}(\mathrm{d} u) .
$$

Thus, $\Psi_{(u)}(A)=0$ for all $u \in \mathbb{R}$ if and only if $\Psi(A)=0$.

Proof of Proposition 2.7. The proof follows from Lemma 4.1 and Lemma 4.2 by taking $A$ to be the set of all point configurations $\beta \in \mathbb{M}$ such that $\beta(\mathbb{R})=\infty$ (in the subcritical case) or $\beta(\mathbb{R})<\infty$ (otherwise).

To prepare for the proof of Proposition 2.8, we need the following lemma.

Lemma 4.3. Let $\varphi(\lambda)=0, \lambda>0$, and $\varphi^{\prime}(\lambda)>0$. If $\chi$ is critical or supercritical, then $\int_{\mathbb{R}} \mathrm{e}^{r u} \Gamma(\mathrm{d} u)<\infty$ a.s. for every $r>r_{0}$, where $r_{0} \geq 0$ is the smallest solution of the equation $\varphi(r)=\varphi^{\prime}(\lambda) r$. 
Proof. By Lemma 4.2 we need to show that $\int_{\mathbb{R}} \mathrm{e}^{r u} \rho(\mathrm{d} u)<\infty$ a.s. for all $r>r_{0}$. Let $\mathcal{A}_{\xi}$ be the $\sigma$-algebra generated by $\left\{\xi_{n}: n \in \mathbb{N}\right\}$. Denote by $J_{(u)}$ the intensity of the point process $\chi_{(u)}, u \in \mathbb{R}$. Fix $\varepsilon>0$. We have $S_{n} / n \rightarrow \varphi^{\prime}(\lambda)$ by the law of large numbers. It follows that, with probability $1, S_{n} / n>\varphi^{\prime}(\lambda)-\varepsilon$ for sufficiently large $n$. Hence,

$$
\begin{aligned}
\mathrm{E}\left[\int_{\mathbb{R}} \mathrm{e}^{r u} \rho(\mathrm{d} u) \mid \mathcal{A}_{\xi}\right] & =1+\sum_{n=1}^{\infty} \int_{\mathbb{R}} \mathrm{e}^{r u} T_{-S_{n}}\left(J^{[n-1]} * J_{\left(\xi_{n}\right)}\right)(\mathrm{d} u) \\
& =1+\sum_{n=1}^{\infty} \int_{\mathbb{R}} \mathrm{e}^{r\left(u-S_{n}\right)}\left(J^{[n-1]} * J_{\left(\xi_{n}\right)}\right)(\mathrm{d} u) \\
& =1+\sum_{n=1}^{\infty} \mathrm{e}^{-r S_{n}} \mathrm{e}^{(n-1) \varphi(r)} Y_{n} \\
& \leq B+\mathrm{e}^{\varphi(r)} \sum_{n=1}^{\infty} \mathrm{e}^{\left(-r \varphi^{\prime}(\lambda)+\varphi(r)+r \varepsilon\right) n} Y_{n},
\end{aligned}
$$

where $Y_{n}=\int_{\mathbb{R}} \mathrm{e}^{r u} J_{\left(\xi_{n}\right)}(\mathrm{d} u)$ and $B$ is an a.s. finite $\mathcal{A}_{\xi}$-measurable random variable. Let us show that $\mathrm{E}\left[Y_{n}\right]$ is finite. Recall that the distribution of $\xi_{n}$ is $\mathrm{e}^{\lambda z} J(\mathrm{~d} z)$. Using the properties of the Palm measures, we have

$$
\begin{aligned}
\mathrm{E}\left[Y_{n}\right] & =\int_{\mathbb{R}}\left(\int_{\mathbb{R}} \mathrm{e}^{r u} J_{(z)}(\mathrm{d} u)\right) \mathrm{e}^{\lambda z} J(\mathrm{~d} z) \\
& =\int_{\mathbb{R}} \mathrm{E}\left[\int_{\mathbb{R}} \mathrm{e}^{r u+\lambda z} \chi_{(z)}(\mathrm{d} u)\right] J(\mathrm{~d} z) \\
& =\mathrm{E}\left[\int_{\mathbb{R}} \int_{\mathbb{R}} \mathrm{e}^{r u+\lambda z} \chi(\mathrm{d} u) \chi(\mathrm{d} z)\right] \\
& =\mathrm{E}\left[\left(\int_{\mathbb{R}} \mathrm{e}^{r u} \chi(\mathrm{d} u)\right)\left(\int_{\mathbb{R}} \mathrm{e}^{\lambda z} \chi(\mathrm{d} z)\right)\right] .
\end{aligned}
$$

By the same estimate as in (3.5), $\mathrm{E}\left[Y_{n}\right]$ is finite. If $\varepsilon>0$ is chosen such that $\varepsilon<\varphi^{\prime}(\lambda)-\varphi(r) / r$ then the series on the right-hand side of (4.2) converges a.s. It follows that

$$
\mathrm{E}\left[\int_{\mathbb{R}} \mathrm{e}^{r u} \rho(\mathrm{d} u) \mid \mathcal{A}_{\xi}\right]<\infty \text { a.s. }
$$

Hence, $\int_{\mathbb{R}} \mathrm{e}^{r u} \rho(\mathrm{d} u)<\infty$ a.s.

We are now in position to prove Proposition 2.8. Let $\sum_{i \in \mathcal{I}} \delta_{P_{i}}$ be a realization of the point process $\Gamma$ from Proposition 2.6. Consider a branching population $\left\{\gamma_{n}: n \in \mathbb{N}_{0}\right\}$ starting with $\Gamma$. That is, let $\left\{\chi_{n}^{(i)}: n \in \mathbb{N}_{0}\right\}, i \in \mathcal{L}$, be independent copies of the branching random walk $\left\{\chi_{n}: n \in \mathbb{N}_{0}\right\}$. Then, $\gamma_{n}=\sum_{i \in \mathcal{X}} T_{P_{i}}\left(\chi_{n}^{(i)}\right)$. We need to show that

$$
\lim _{n \rightarrow \infty} \max \gamma_{n}=-\infty \quad \text { a.s. }
$$

Let $\mathcal{A}_{\Gamma}$ be the $\sigma$-algebra generated by $\Gamma$. We have

$$
\mathrm{P}\left[P_{i}+\max \chi_{n}^{(i)}>-\varepsilon n \mid \mathcal{A}_{\Gamma}\right] \leq \mathrm{e}^{-n I\left(-\varepsilon-P_{i} / n\right)} \leq \mathrm{e}^{-n I(-\varepsilon)} \mathrm{e}^{I^{\prime}(-\varepsilon) P_{i}} .
$$


We have $I(-\varepsilon)>0$ and $I^{\prime}(-\varepsilon)>r_{0}$ if $\varepsilon>0$ is sufficiently small since $\lim _{\varepsilon \rightarrow 0} I(-\varepsilon)=$ $I(0)=-\inf _{t \in \mathbb{R}} \varphi(t)>0$ and $\lim _{\varepsilon \rightarrow 0} I^{\prime}(-\varepsilon)=I^{\prime}(0)=\arg \min \varphi>r_{0}$. It follows from Lemma 4.3 that

$$
\sum_{n=1}^{\infty} \mathrm{P}\left[\max \gamma_{n}>-\varepsilon n \mid \mathcal{A}_{\Gamma}\right]<\infty \text { a.s. }
$$

An application of the Borel-Cantelli lemma completes the proof of (4.3).

\section{Proofs of Theorem 2.1 and Proposition 2.5}

The proof is based on the general results of [17]. By Theorem 2.3.6 of [17], a measure $v$ on $\mathbb{R}^{d}$ which is finite on bounded sets is an intensity of some equilibrium state if and only if the branching population starting from a Poisson point process with intensity $v$ converges weakly to a point process with intensity $v$. (In [17], such measures $v$ are called stable.) Thus, by Theorem 1.1, the intensity $v$ of an equilibrium state $\Xi$ is a linear combination with nonnegative coefficients of the measures of the form $e_{\lambda}$, where $\lambda \in K_{\mathrm{st}}$.

By Theorem 4.7.28 of [17], any equilibrium state $\Xi$ with intensity $v$ is a mixture of the form (2.3) if the following two conditions (called aperiodicity and regularity) are satisfied.

1. For every probability measure $\sigma$ on $\mathbb{R}$ which is absolutely continuous with respect to the Lebesgue measure, we have

$$
\lim _{n \rightarrow \infty}\left\|\sigma * D^{[n]}-\sigma * D^{[n+1]}\right\|=0
$$

where $D$ is the probability measure defined in (3.1) and $\|\cdot\|$ denotes the total variation.

2. For every bounded Borel set $A \subset \mathbb{R}$ and every $\varepsilon>0$,

$$
\lim _{n \rightarrow \infty} v\left\{z \in \mathbb{R}: J^{[n]}(A-z)>\varepsilon\right\}=0 .
$$

See Proposition 3.8.4 of [17] for the first condition and Theorem 4.7.19 of [17] for the second condition. The aperiodicity condition holds as long as the measure $J$ is nonlattice by Theorem 11.10.4 and Proposition 11.9.1 of [21]. Let us verify the regularity condition. In the subcritical case the measure on the left-hand side of (5.1) is 0 for sufficiently large $n$. In the nonsubcritical case, we need a different argument. Without restriction of generality, let $v=e_{\lambda}$, where $\varphi(\lambda)=0, \lambda>0$, and $\varphi^{\prime}(\lambda)>0$. It suffices to consider the case $A=(a, \infty)$, where $a \in \mathbb{R}$. We have, for every $t \geq 0$,

$$
J^{[n]}(A-z) \leq \mathrm{e}^{-t(a-z)} \int_{\mathbb{R}} \mathrm{e}^{t u} J^{[n]}(\mathrm{d} u)=\mathrm{e}^{-t(a-z)+n \varphi(t)} .
$$

It follows that

$$
\left\{z \in \mathbb{R}: J^{[n]}(A-z)>\varepsilon\right\} \subset\left(-\frac{\varphi(t)}{t} n+\frac{\log \varepsilon}{t}+a, \infty\right) .
$$

We can choose $t>0$ such that $\varphi(t)<0$. For example, take $t=\lambda-\delta$ for sufficiently small $\delta>0$. The $e_{\lambda}$-measure of the set on the right-hand side of (5.2) goes to 0 as $n \rightarrow \infty$. This completes the proof of Theorem 2.1. Proposition 2.5 follows from the regularity condition (5.1) by Theorem 4.7.19 of [17]. 


\section{References}

[1] AïdÉkon, E., Berestycki, J., Brunet, É. AND Shi, Z. (2011). The branching Brownian motion seen from its tip. Preprint. Available at http://arxiv.org/abs/1104.3738v2.

[2] Arguin, L.-P., Bovier, A. And Kistler, N. (2011). The extremal process of branching Brownian motion. Preprint. Available at http://arxiv.org/abs/1103.2322v1.

[3] Athreya, K. B. And Ney, P. E. (1972). Branching Processes. Springer, New York.

[4] Biggins, J. D. (1976). The first- and last-birth problems for a multitype age-dependent branching process. Adv. Appl. Prob. 8, 446-459.

[5] Biggins, J. D. (1977). Chernoff's theorem in the branching random walk. J. Appl. Prob. 14, 630-636.

[6] Bramson, M. D. (1978). Maximal displacement of branching Brownian motion. Commun. Pure Appl. Math. 31, 531-581.

[7] Brown, B. M. and Resnick, S. I. (1977). Extreme values of independent stochastic processes. J. Appl. Prob. 14, 732-739.

[8] Brunet, E. And Derrida, B. (2009). Statistics at the tip of a branching random walk and the delay of traveling waves. Preprint. Available at http://arxiv.org/abs/0905.0613v1.

[9] Brunet, E. ANd Derrida, B. (2010). A branching random walk seen from the tip. Preprint. Available at http://arxiv.org/abs/1011.4864v1.

[10] Deny, J. (1960). Sur l'équation de convolution $\mu=\mu * \sigma$. Séminaire Brelot-Choquet-Deny Theorie du Potentiel 4, $11 \mathrm{pp}$.

[11] Gorostiza, L. G. and Wakolbinger, A. (1991). Persistence criteria for a class of critical branching particle systems in continuous time. Ann. Prob. 19, 266-288.

[12] Ivanoff, G. (1980). The branching random field. Adv. Appl. Prob. 12, 825-847.

[13] Kabluchio, Z. (2010). Stationary systems of Gaussian processes. Ann. Appl. Prob. 20, 2295-2317.

[14] Kabluchko, Z., Schlather, M. and De HaAn, L. (2009). Stationary max-stable fields associated to negative definite functions. Ann. Prob. 37, 2042-2065.

[15] Kallenberg, O. (1977). Stability of critical cluster fields. Math. Nachr. 77, 7-43.

[16] Lalley, S. P. AND Sellke, T. (1987). A conditional limit theorem for the frontier of a branching Brownian motion. Ann. Prob. 15, 1052-1061.

[17] Liemant, A., Matthes, K. and Wakolbinger, A. (1988). Equilibrium Distributions of Branching Processes (Math. Appl. 34). Kluwer, Dordrecht.

[18] Lyons, R., Pemantle, R. and Peres, Y. (1995). Conceptual proofs of $L \log L$ criteria for mean behavior of branching processes. Ann. Prob. 23, 1125-1138.

[19] Madaule, T. (2011). Convergence in law for the branching random walk seen from its tip. Preprint. Available at http://arxiv.org/abs/arXiv:1107.2543v2.

[20] MaillaRD, P. (2011). A characterisation of superposable random measures. Preprint. Available at http://arxiv.org/ abs/1102.1888v1.

[21] Matthes, K., Kerstan, J. And Mecke, J. (1978). Infinitely Divisible Point Processes. John Wiley, Chichester.

[22] Ruzmaikina, A. and Aizenman, M. (2005). Characterization of invariant measures at the leading edge for competing particle systems. Ann. Prob. 33, 82-113.

[23] Wakolbinger, A. (1994). Poblaciones Aleatorias Ramificadas y sus Equilibrios, (Math. Contrib. Res. Notes 9). Sociedad Matemática Mexicana, México.

[24] WaKolbinger, A. (1995). Limits of spatial branching populations. Bernoulli 1, 171-185. 